\title{
STAR RNA-binding protein Quaking suppresses cancer via stabilization of specific miRNA
}

\author{
An-Jou Chen, ${ }^{1,2,3}$ Ji-Hye Paik, ${ }_{1,2,3}^{1,3 a i l e i}$ Zhang, ${ }^{1,2}$ Sachet A. Shukla, ${ }^{1,2}$ Richard Mortensen, ${ }^{4}$ \\ Jian $\mathrm{Hu}^{1,2,3,5}$ Haoqiang Ying, ${ }^{1,2,3,5}$ Baoli Hu, ${ }^{1,2,5}$ Jessica Hurt, ${ }^{6}$ Natalie Farny, ${ }^{6}$ Caroline Dong, ${ }^{1,2}$ \\ Yonghong Xiao, ${ }^{1,2}$ Y. Alan Wang, ${ }^{1,2,5}$ Pamela A. Silver, ${ }^{6}$ Lynda Chin, ${ }^{1,2,5,7}$ Shobha Vasudevan, ${ }^{4}$ \\ and Ronald A. DePinho ${ }^{1,2,3,8,9}$ \\ ${ }^{1}$ Belfer Institute for Applied Cancer Science, ${ }^{2}$ Department of Medical Oncology, Dana-Farber Cancer Institute, Harvard Medical \\ School, Boston, Massachusetts 02115, USA; ${ }^{3}$ Department of Medicine and Genetics, Harvard Medical School, Boston, \\ Massachusetts 02115, USA; ${ }^{4}$ Cancer Center, Massachusetts General Hospital, Harvard Medical School, Boston, Massachusetts \\ 02129, USA; ${ }^{5}$ Department of Genomic Medicine, University of Texas MD Anderson Cancer Center, Houston, Texas 77030, USA; \\ ${ }^{6}$ Department of Systems Biology, Harvard Medical School, Boston, Massachusetts 02115, USA; ${ }^{7}$ Department of Dermatology, \\ Brigham and Women's Hospital, Harvard Medical School, Boston, Massachusetts 02115, USA; ${ }^{8}$ Department of Cancer Biology, \\ University of Texas MD Anderson Cancer Center, Houston, Texas 77030, USA
}

Multidimensional cancer genome analysis and validation has defined Quaking (QKI), a member of the signal transduction and activation of RNA (STAR) family of RNA-binding proteins, as a novel glioblastoma multiforme (GBM) tumor suppressor. Here, we establish that p53 directly regulates QKI gene expression, and QKI protein associates with and leads to the stabilization of miR-20a; miR-20a, in turn, regulates TGF $\beta R 2$ and the TGF signaling network. This pathway circuitry is substantiated by in silico epistasis analysis of its components in the human GBM TCGA (The Cancer Genome Atlas Project) collection and by their gain- and loss-of-function interactions in in vitro and in vivo complementation studies. This p53-QKI-miR-20a-TGF $\beta$ pathway expands our understanding of the $\mathbf{p} 53$ tumor suppression network in cancer and reveals a novel tumor suppression mechanism involving regulation of specific cancer-relevant microRNAs.

[Keywords: cancer; GBM; miRNA; p53; TGF $\beta$; QKI]

Supplemental material is available for this article.

Received February 4, 2012; revised version accepted May 24, 2012.

MicroRNAs (miRNAs), small noncoding RNAs that bind specific messenger RNAs (mRNAs) and regulate their translation and/or their stability, have been linked to cancer pathogenesis of virtually all known cancer pathways and are subject to recurrent genomic alteration in human cancer and to aberrant transcriptional regulation by key cancer genes such as MYC and TP53 (Calin et al. 2002; Dews et al. 2006; Esquela-Kerscher and Slack 2006; He et al. 2007b; Sachdeva et al. 2009). The Cancer Genome Atlas Project (TCGA) (The Cancer Genome Atlas Research Network 2008) has compiled a large collection of highresolution genomic data from clinically annotated tumor samples, providing an unprecedented framework for the rapid discovery of novel cancer genes guided by cancer gene alterations across multiple dimensions of the genome. Here, in the course of conducting a functional genomic

${ }^{9}$ Corresponding author

E-mail rdepinho@mdanderson.org

Article is online at http://www.genesdev.org/cgi/doi/10.1101/gad.189001.112. screen for p53 targets and mining the TCGA glioblastoma multiforme (GBM) data set and others (Mulholland et al. 2006; Wiedemeyer et al. 2008; Yin et al. 2009), we identified Quaking (QKI) as a potential tumor suppressor gene that is frequently deleted in GBM. The potential importance of QKI in GBM pathogenesis is further elevated by its direct regulation by the TP53 tumor suppressor, the most commonly mutated gene in primary GBM (Zheng et al. 2008).

QKI is an RNA-binding protein that belongs in the signaling transduction and activation of RNA (STAR) family of proteins (Vernet and Artzt 1997). The QKI gene generates alternative transcripts that encode three isoforms-QKI-5, QKI-6, and QKI-7-that share an RNAbinding $\mathrm{KH}$ domain but differ by several amino acids at the $\mathrm{C}$ terminus. QKI isoforms can dimerize with one another and shuttle between the nucleus and the cytoplasm (Chen and Richard 1998; Wu et al. 1999). QKI appears to be relevant to brain biology, as evidenced by its underexpression in GBM (Li et al. 2002) and in schizophrenia (Aberg et al. 2006; Haroutunian et al. 2006) and its capacity to bind (directly or 
indirectly) and regulate mRNA of myelin genes, $M B P$ (Larocque et al. 2002), MAG (Wu et al. 2002), and the myelin-differentiating gene $p 27$ (Larocque et al. 2005), among others (Lobbardi et al. 2011; Zearfoss et al. 2011). A spontaneous germline $\sim 1-\mathrm{Mb}$ deletion of the QKI promoter/enhancer region that eliminates QKI-6 and QKI-7 isoform expressions in oligodendrocytes (yet maintains expressions in astrocytes) produces myelin deficiency and a whole-body "quaking" phenotype in mice (Sidman et al. 1964; Ebersole et al. 1996). QKI nullizygosity is embryonic-lethal in mice due to defective vasculature development (Li et al. 2003).

In this study, we validate $Q K I$ as a potent GBM tumor suppressor, document $Q K I$ transcriptional regulation by p53, and establish QKI-directed stabilization of miR-20a and associated alterations in the miR-20a target TGF $\beta$ R2. The capacity of p53-regulated QKI to modulate miR-20a stability defines a new pathway of tumor suppression involving a novel mechanism of regulated stabilization of specific miRNAs in human cancer.

\section{Results}

QKI is a frequently deleted tumor suppressor gene in $G B M$

The QKI gene emerged from a combined in silico and functional screen designed to identify potential p53responsive genes that are up-regulated in the setting of DNA damage response to short telomeres (JH Paik and RA DePinho, unpubl.; see below). As a first step in prioritizing such putative p53 targets for further study, their potential cancer relevance was determined through examination of various cancer genome data sets. These analyses revealed strikingly frequent focal deletions of chromosome 6q26-27, which includes $Q K I$, in human primary GBM that are present in several of our array-CGH data sets (Maher et al. 2006; Wiedemeyer et al. 2008; Yin et al. 2009). Subsequent analysis of the extensive multidimensional TCGA data sets showed loss of QKI expression in 92 out of $420(22 \%)$ transcriptional profiles, deletion of QKI in 85 out of $420(20 \%)$ array-CGH profiles, and QKI methylation (chromosome 6 , base $163,755,107)$ in 50 out of $250(20 \%$ ) methylation profiles; mutation data for QKI have not been completed. The minimum common region at $6 \mathrm{q} 26-27$ is defined by a focal, hemizygous deletion of three genes-QKI, $P A C R G$, and PARK2 (Supplemental Fig. 1A) -with several samples containing deletion of $Q K I$ only (Supplemental Fig. 1B). Of these three genes, only the expression of QKI and PARK2 correlates with copy number (Supplemental Fig. $1 \mathrm{C}$ ), suggesting that $Q K I$ and PARK2 are both cancer-relevant targets of the 6q26-27 deletion event. Furthermore, QKI expression and methylation negatively correlate (Supplemental Fig. 1D,E), suggesting that both QKI deletion and methylation events can drive lower QKI expression.

These genomic and expression data on QKI prompted extensive gain- and loss-of-function validation studies to assess whether QKI functions as a glioma tumor suppressor in both human and mouse model systems. In the loss-of-function studies, we used QKI-expressing human GBM cell lines U87 and Hs683 and two independent QKI shRNAs with $>50 \%$ knockdown efficiency of all three QKI transcript isoforms. The effective QKI shRNAs (shQKI-1 and shQKI-2) resulted in increased soft agar colony formation relative to GFP shRNA (shGFP) and an ineffective QKI shRNA control (shQKI-3) (Fig. 1A,B). Similarly, two independent murine Qki shRNAs increased soft agar colony formation in Qki-expressing primary mouse Ink4a/Arf ${ }^{-1-} \mathrm{Pten}^{-1-}$ astrocytes; i.e., a primary cell system engineered with two signature mutations found in human GBM (Fig. 1C; Bachoo et al. 2002). In tumorigenesis assays, QKI knockdown enabled tumor formation with the poorly tumorigenic human Hs683 cell line and nontumorigenic primary mouse Ink $4 a / \mathrm{Arf}^{-1-} \mathrm{Pten}^{-/-}$ astrocytes (Fig. 1D).

Since PARK2 is a recently reported tumor suppressor gene in GBM (Veeriah et al. 2010), and PARK2 and QKI reside in the same deletion on chromosome 6q26-27, we next examined whether PARK2 and QKI cooperate with each other in GBM tumor suppression. We observed that knocking down PARK2 or QKI individually increased soft agar colony formation, while knocking down PARK2 and $Q K I$ together further enhanced in vitro tumorigenicity (Supplemental Fig. 1F,G). Our result suggests that while PARK2 and QKI each exert tumor-suppressive activity, their combined deletion on chromosome 6q26-27 cooperates to drive tumorigenesis.

In the gain-of-function studies, reconstitution of QKI in the low-expressing human GBM cell lines LN18 and U343 resulted in decreased soft agar colony formation (Fig. 1E). Notably, transduction of QKI mutant (V157E) isoforms, which abrogates QKI RNA binding (Larocque et al. 2002), enhanced in vitro oncogenic activity as measured by soft agar colony formation (Fig. 1E), thus establishing that QKI exerts its tumor-suppressive effects via its RNA regulatory functions. Together, the TCGA genomic data, coupled with cross-species functional validation in vitro and in vivo, support a tumor suppressor role for QKI in GBM.

\section{QKI is a TP53 target gene}

The discovery of QKI in a p53-dependent target screen prompted an assessment of whether QKI is a direct transcriptional target of the p53 tumor suppressor on several levels. First, we used the cell system in which QKI was first identified: $\mathrm{mTerc}^{-1-} \mathrm{Atm}^{-/-} \mathrm{p}^{-1-}$ mouse embryonic fibroblasts (MEFs) (Maser et al. 2007) harboring a TP53-estrogen receptor (p53ER) (Christophorou et al. 2005) expression vector that can be activated upon tamoxifen treatment. Tamoxifen treatment of these cells resulted in increased endogenous Qki-5 and Qki-7 mRNA expression (Fig. 2A) and p21 mRNA expression (Fig. 2B); the Qki-6 mRNA level, which was not detectable in mTerc ${ }^{-1-} \mathrm{Atm}^{-1-}$ p53 ${ }^{-1-}$ MEFs, was expressed and upregulated in wild-type human astrocytes exposed to doxorubicin (see Supplemental Fig. 3). Increased Qki protein levels tracked with p53-directed Qki transcriptional activation in the same cell system (Fig. 2C). Second, using 
A.

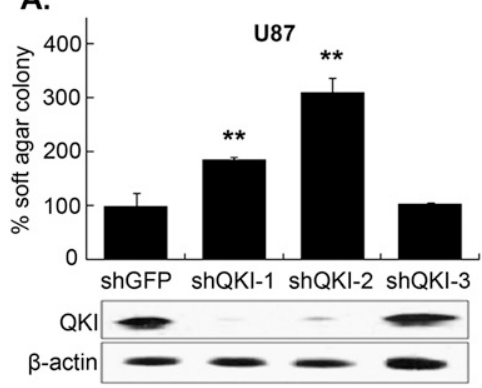

C.

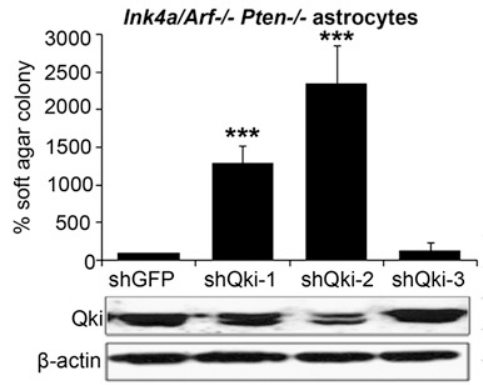

B.

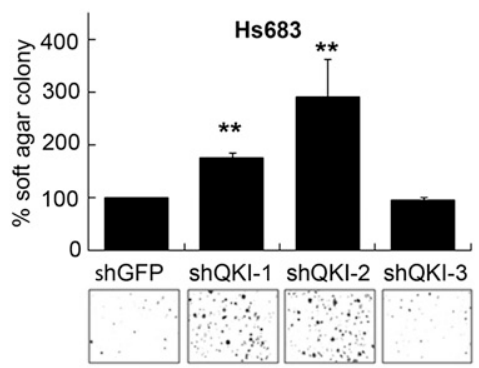

D.

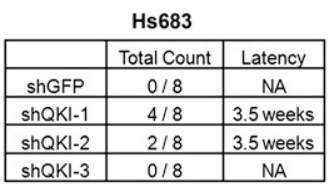

Ink4a/Arf-/- Pten-/- astrocytes

\begin{tabular}{|c|c|c|}
\hline & Total Count & Latency \\
\hline shGFP & $0 / 10$ & NA \\
\hline shQki-1 & $1 / 10$ & 8 weeks \\
\hline shQki-2 & $7 / 10$ & 7.5 weeks \\
\hline shQki-3 & $0 / 10$ & NA \\
\hline
\end{tabular}

E.
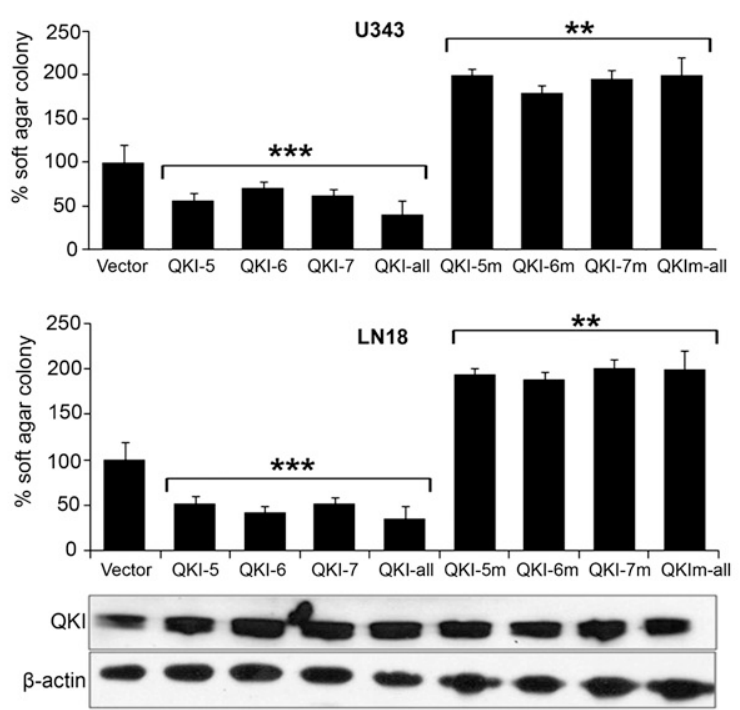

Figure 1. $Q K I$ is a GBM tumor suppressor gene. $(A-C)$ QKI was knocked down by shRNAs, and 5000 human GBM cells or 20,000 mouse astrocytes were plated per well in six-well plates for soft agar colony assay. Experiments were performed in triplicates. U87 (p53-WT) $(A)$ and Hs683 (p53-null) (B) human GBM cell lines, as well as Ink4a/Arf ${ }^{-1-}$ Pten $^{-1-}$ primary mouse astrocytes $(C)$ transduced with two independent shRNAs (shQKI-1 and shQKI-2) against QKI showed increased soft agar colony growth relative to shGFP and noneffective shQKI control (shQKI-3). A Western blot showing QKI protein levels upon shRNA knockdown is presented in $A$ for the human system and in $C$ for the mouse system. The soft agar colony result is presented in $B$; magnification $40 \times$. $\left(^{\star \star}\right) P<0.01 ;\left(^{\star \star \star}\right) P<0.001$ compared with shGFP control. (D) We injected $2 \times 10^{6} \mathrm{Hs} 683$ cells or Ink4a) Arf $^{-1-}$ Pten $^{-1-}$ mouse astrocytes with QKI knockdown by two independent shRNAs into each flank of immuno-compromised nude mice. shQKI cells in both cell types induced subcutaneous tumor formation in immuno-compromised nude mice but not the shGFP and noneffective shQKI controls. (E) Wild-type (WT) or RNA-binding-deficient (V157E) QKI isoforms were overexpressed individually or all together in U343 (p53-WT) and LN18 (p53-null) human GBM cells, and 5000 cells per well in six-well plates were plated for soft agar colony assay. Experiments were performed in triplicates. Overexpression of QKI-5, QKI-6, and QKI-7 isoforms in U343 and LN18 cells decreased soft agar colony formation. Overexpression of mutant QKI isoforms increased soft agar colony formation as a dominant-negative effect. QKI-5 and QKI-5 mutant migrate at $\sim 45 \mathrm{kDa}, \mathrm{QKI}-6$ and QKI-6 mutant migrate at $\sim 38 \mathrm{kDa}, \mathrm{QKI}-7$ and QKI-7 mutant migrate at $\sim 42 \mathrm{kDa}$. U343 cells were seeded in soft agar containing $5 \%$ serum; LN18 cells were in $1 \%$ serum. $\left.\left(^{\star \star}\right) P<0.01 ;{ }^{\star \star \star}\right) P<0.001$ compared with vector control. primary mouse Ink $4 a / A r f^{-1-}$ and $p 53^{-1-}$ astrocytes, we confirmed that DNA damage-induced activation of endogenous p53 (with doxorubicin) also resulted in increased Qki protein expression only in Ink4a/Arf ${ }^{-1-}$ cells (Fig. 2D). Similarly, adenoviral p53 transduction resulted in elevated Qki protein levels in $p 53^{-/-}$astrocytes (Fig. 2E). Finally, upon doxorubicin treatment of wild-type human astrocytes, chromatin immunoprecipitation (ChIP) assays for TP53 demonstrated significant enrichment of a $p 21$ promoter fragment as well as two QKI promoter elements harboring putative TP53-binding sites, relative to the IgG controls (Fig. 2F). Thus, we conclude that p53 physically binds to the $Q K I$ promoter and up-regulates endogenous QKI gene expression in the setting of activated p53.

\section{QKI and miR-20a modulate cancer-relevant signaling networks}

To understand the mechanism of $Q K I$ as a GBM tumor suppressor gene, we performed transcriptional profiling and knowledge-based analysis to identify potential QKIregulated mRNAs and linked pathways. We compared the transcriptional profiles of shGFP- and shQKI-transduced human Hs683 cells and further intersected these profiles with those of shQki- and shGFP-transduced Ink4a/Arf ${ }^{-1-}$ Pten $^{-1-}$ primary mouse astrocytes. For these cross-species studies, transcriptional profiles were obtained using two different QKI-directed shRNAs for both the human and mouse systems. The 219 human and 98 
A.

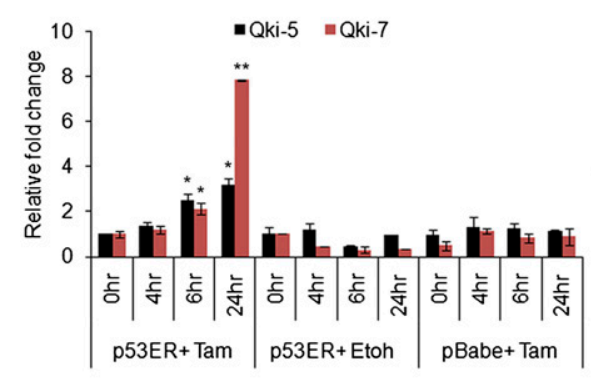

c.

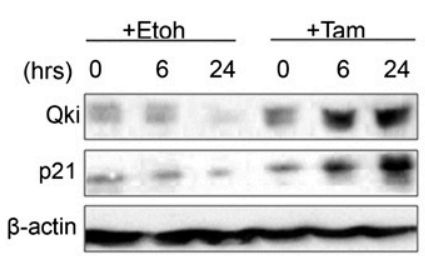

E.

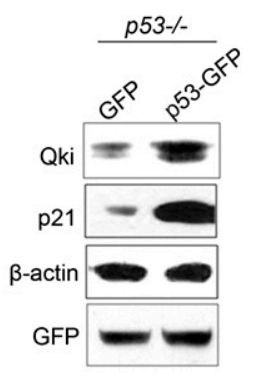

B.

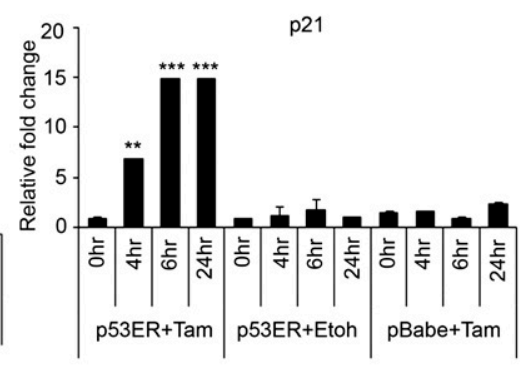

D.

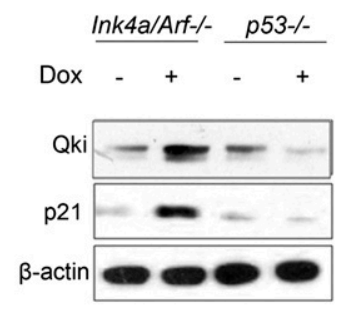

F.

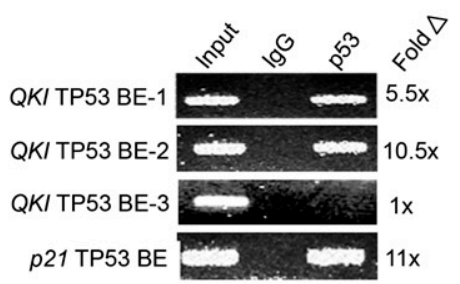

Figure 2. QKI is a p53 target gene. $(A-C)$ Qki was originally identified in $\mathrm{mTerc}^{-1-} \mathrm{Atm}^{-/-}$ $p 53^{-/-}$MEFs transduced with the inducible p53ER expression vector. To verify, Qki RNA $(A)$ and protein $(C)$ levels were measured and confirmed to be activated upon tamoxifen (Tam) treatment relative to ethanol (Etoh); p21 $(B)$ served as positive control. RNA level was measured by qPCR. $\left(^{\star}\right) P<0.05$; $\left(^{\star *}\right) P<$ $\left.0.01 ;{ }^{\star \star \star}\right) P<0.001$ compared with 0 h. $(D)$ Ink $4 a /$ Arf $^{-1-}$ and $p 53^{-1-}$ mouse astrocytes were treated with doxorubicin or water. Upon DNA damage agent doxorubicin (Dox) treatment, Qki expression was enhanced in Ink4a/ $\mathrm{Arf}^{-1-}$ mouse astrocytes due to p53 activation, but not in $p 53^{-1-}$ mouse astrocytes. $(E)$ Adenovirus p53-GFP was transduced into $p 53^{-1-}$ mouse astrocytes for $24 \mathrm{~h}$. Qki and p21 protein levels were elevated in $p 53^{-/-}$ mouse astrocytes transduced with adenovirus p53-GFP relative to adenovirus GFP control. $(F)$ Human astrocytes were treated with doxorubicin, and p53 ChIP analysis was performed. Upon doxorubicin treatment, p53 bound to two QKI promoter elements (BE) in normal human astrocytes by ChIP analysis; p21 was used as positive control. Fold changes between p53 and IgG as measured by qPCR are listed at the right of each panel. mouse genes with $\geq 1$.5-fold change (Supplemental Table 1) centered on TGF $\beta$ and ERK networks as determined by Ingenuity Pathway Analysis (IPA) (Supplemental Fig. 2A), which were confirmed by RT-qPCR and biochemical studies (see below).

In addition to transcriptional profiling, we also performed miRNA profiles with the Exiqon version 11.0 miRCURY LNA miRNA platform containing 1300 human and 900 mouse miRNA genes. Specifically, 30 miRNA genes were reproducibly altered by $\geq 1.5$-fold change in human U87 cells, 37 were altered in human Hs683 cells, and nine were altered in Ink4a/Arf ${ }^{-1-}$ Pten $^{-1-}$ mouse astrocytes (Supplemental Table 2). Confirmatory RT-qPCR was performed for the seven miRNAs that were altered in at least two of the three cell model systems, which demonstrated that miR-20a was the only miRNA that was consistently down-regulated in all three cell systems (Supplemental Fig. 2B). In addition, progressive reduction of Qki expression by increasing concentration of siRNA against Qki tracked closely with decreasing miR-20a levels in Ink4a/Arf ${ }^{-1-}$ Pten $^{-1-}$ astrocytes-but not miR-18a, an miRNA that resides in the same miR-17-92 cluster as miR-20a (Supplemental Fig. 2C)-further supporting a QKI-miR-20a link. Then, to examine whether QKI affects the Drosha-regulated primary miRNA (primiRNA) and Dicer-regulated precursor miRNA (premiRNA) levels for miR-20a, we measured their levels in both QKI knockdown and overexpression systems. We did not observe a change in pri-miR-20a or pre-miR-20a levels in QKI knockdown Hs683 cells and Ink4a/Arf ${ }^{-1-}$ Pten $^{-1-}$ astrocytes (Supplemental Fig. 2D,E). Furthermore, while there were increased mature miR20a levels in QKI overexpressing LN18 and U343 GBM cells, we did not observe a change in pre-miR-20a or pri-miR-20a levels (Supplemental Fig. 2F,G), suggesting that QKI only affects the mature miR-20a levels. While it is possible that QKI regulates a number of miRNAs, the consistent dependency of mature miR-20a levels on QKI prompted further in-depth analysis of this miRNA and its function in GBM.

miR-20a belongs to miR-17-92, an miRNA cluster that resides in intron 3 of the MIR17HG gene at human chromosome 13q31.3. Corresponding well with the above transcriptome IPA findings, transcriptomic analysis of QKI knockdown U87, Hs683, and Ink4a/Arf ${ }^{-1-}$ Pten $^{-1-}$ 
mouse astrocytes stably transduced with miR-20a or a scrambled nontargeting miRNA (miR-NT) (Supplemental Fig. $2 \mathrm{H}$ ) showed TGF $\beta$ and ERK to be the most highly altered pathways among transcripts exhibiting a $\geq 1.5$-fold change (Supplemental Fig. 2I). Specifically, 234 transcripts were altered in U87 cells, 91 were altered in Hs683 cells, and 66 were altered in Ink4a/Arf ${ }^{-1-}$ Pten $^{-1-}$ mouse astrocytes (Supplemental Table 3). As observed with the QKI knockdown transcriptional profile data (Supplemental Table 1), the three cell systems did not share any overlapping transcripts upon miR-20a overexpression, an observation that was confirmed by RTqPCR of the specific genes representing these pathways in the three cell systems (data not shown). These results suggest that the actions of QKI knockdown and miR-20a overexpression converge on the regulation of TGF $\beta$ and ERK signaling networks in primary and transformed glial cells.

\section{p53 activates miR-20a}

Next, we sought to determine the mechanistic basis of QKI-directed regulation of miR-20a expression. As QKI was directly activated by p53 (see Fig. 2), we sought to determine whether doxorubicin-induced activation of TP53 in normal human astrocytes was associated with increased QKI and miR-20a levels. We observed increased levels of QKI and miR-20a together with known TP53 target p21 upon doxorubicin treatment, which was not observed in doxorubicin-treated astrocytes transduced with shTP53 (Supplemental Fig. 3A). Human astrocytes transduced with shQKI showed decreased miR-20a levels, although miR-20a levels in shQKI cells were also marginally induced upon doxorubicin treatment, indicating that p53-dependent, QKI-independent mechanisms modestly regulate miR-20a levels (Supplemental Fig. 3A).

To distinguish whether increased miR-20a levels was due to p53-dependent transcriptional activation or QKIdependent up-regulation, we monitored the expression of pre-miR-20a and pri-miR-20a and the expression of the MIR17HG gene (wherein miR-17-92 cluster resides). Doxorubicin treatment increased the levels of mature miR-20a, pre-miR-20a, pri-miR-20a, and MIR17HG mRNA in shGFP- and shQKI-transduced astrocytes but not in shTP53-transduced astrocytes (Supplemental Fig. 3A). Similarly, transfection of pcDNA-p53 (Stommel and Wahl 2004) into $p 53^{-1-}$ mouse astrocytes transduced with shGFP or shQki increased expression of p21, mature miR-20a, premiR-20a, pri-miR-20a, and Mir17hg relative to vectortransfected controls (Supplemental Fig. 3B).

\section{QKI associates with and regulates the stability} of mature miR-20a

To substantiate the functional interaction of QKI and miR20a, we performed protein-RNA immunoprecipitation assays to assess whether endogenous QKI interacts with miR-20a. In Ink $4 a / \mathrm{Arf}^{-1-}$ mouse astrocytes, doxorubicininduced activation of p53 and coupled up-regulation of endogenous Qki levels (see Fig. 2D) resulted in miR-20a association with $\mathrm{Qki}$, which was not detected in non- doxorubicin-treated Ink4a/Arf ${ }^{-1-}$ astrocytes or in $p 53^{-1-}$ mouse astrocytes (Supplemental Fig. 4A,B). Antibody against Sc35, an RNA-binding protein known to regulate mRNA splicing, served as a negative control and did not pull down miR-20a (Supplemental Fig. 4A,B). As a control, we also did not observe QKI pulling down miR-18a (Supplemental Fig. 4A,B). In contrast, Qki did not coimmunoprecipitate pre-miR-20a or pri-miR-20a, whereas anti-Sc35 immunoprecipitated pri-miR-20a and Sc35 mRNA (Supplemental Fig. 4A,B), agreeing with published reports that Sc35 protein colocalizes with microprocessor machineries and may be involved in regulating primary miRNA processing (Kataoka et al. 2009).

Next, to test whether QKI can associate with miR-20a, we performed a series of in vivo and in vitro experiments. First, using human astrocytes treated with or without doxorubicin, we performed in vitro UV cross-linking followed by RNase treatment and immunoprecipitation of the endogenous QKI. We observed that in human astrocytes treated with doxorubicin, all QKI isoforms associated with miR-20a but not with pre-miR-20a or pri-miR-20a (Fig. 3A); non-doxorubicin-treated human astrocytes did not show association with miR-20a (Fig. 3A). To test association in vivo, we performed in vivo UV cross-linking upon transiently transfecting V5-tagged QKI-6 and QKI-6 mutant into 293T human embryonic kidney (HEK) cells. We observed that V5-QKI-6 associated with miR-20a more efficiently than with pre-miR-20a or pri-miR-20a in 293T HEK cells; no miRNAs were detected in the V5-QKI-6 mutant sample despite its robust expression and immunoprecipitation (Fig. 3B). Next, we purified V5-tagged QKI6 and RNA-binding-deficient QKI-6 mutant from 293T HEK cells (Supplemental Fig. 4C) for further validation studies. In vitro UV cross-linking with purified proteins and radioactively labeled miRNAs, followed by RNase treatment and immunoprecipitation using V5 beads, showed that purified V5-QKI-6 associated with labeled miR-20a but not with pre-miR-20a, miR-18a, and miR-20a ${ }^{\star}$; V5QKI-6 mutant and antibody control Flag purifications did not demonstrate association with any miRNAs (Fig. 3C). Thus, we conclude that QKI associates with miR-20a via an intact RNA-binding domain. The finding that the QKI $\mathrm{KH}$ domain mutant could not interact with or stabilize miR-20a (Fig. 3) is consistent with a previous study demonstrating a $\mathrm{KH}$ domain requirement for direct interaction of the RNA-binding protein hnRNP E2 with another specific miRNA, miR328 (Eiring et al. 2010).

Since QKI associates with mature miR-20a and not with pre-miR-20a, pri-miR-20a, or miR-20a* (Fig. 3A-C), we considered the possibility that QKI may regulate mature miR-20a stabilization. To test this hypothesis, we monitored miR-20a levels in 293T HEK cells that were transiently transfected with QKI-5, QKI-6, and QKI-7 together and treated with the RNA polymerase II inhibitor $\alpha$-amanitin. We observed miR-20a stabilization in all QKI isoform-transfected cells, extending miR-20a halflife from between 3 and $6 \mathrm{~h}$ to between 9 and $12 \mathrm{~h}$ (Fig. 3D). miR-20a stabilization was not observed in vectortransfected controls or in cells transfected with all three mutant QKI isoforms deficient of RNA-binding abilities 
A.
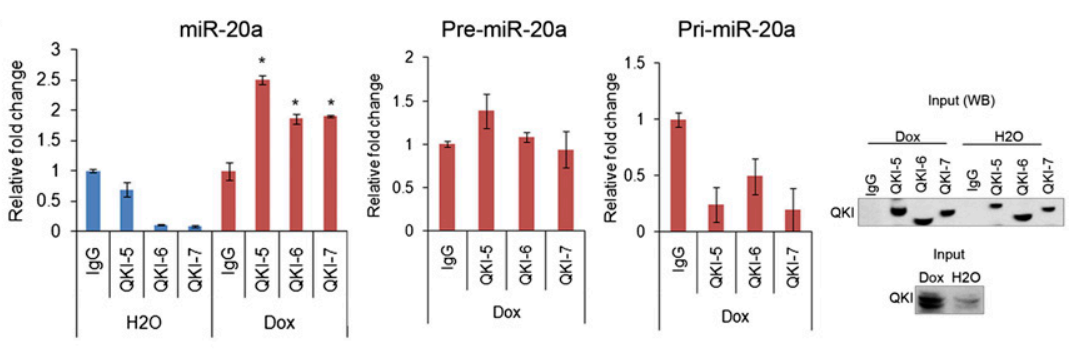

B.

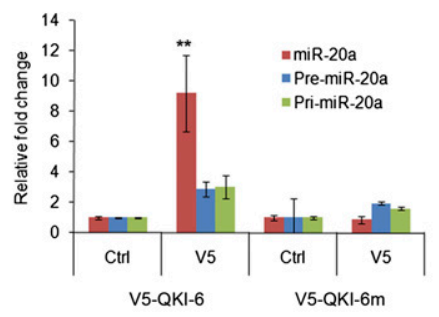

C.

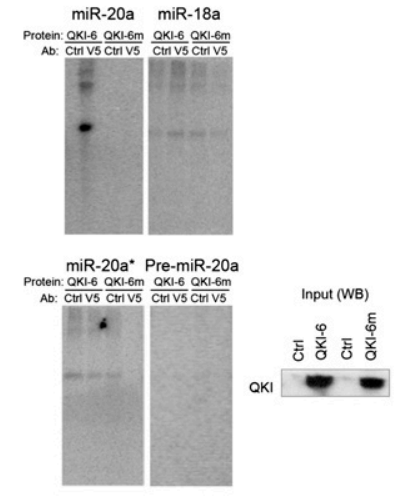

D.
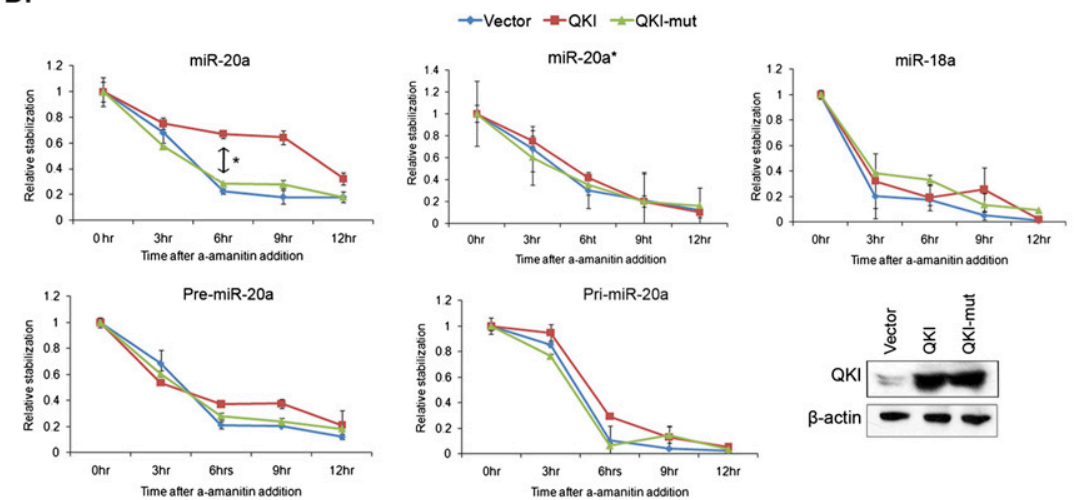

Figure 3. QKI associates with and stabilizes miR-20a. We investigated whether QKI-5, QKI-6, and QKI-7 isoforms each associate with and stabilize miR-20a and focused on QKI-6 for in-depth analysis. QKI6 is the isoform previously shown to have evolutionarily conserved function in Caenorhabditis elegans (Schumacher et al. 2005). $(A)$ In vitro UV cross-linking was performed using normal human astrocytes treated with or without doxorubicin, and QKI-5, QKI-6, or QKI-7 was immunoprecipitated by isoform-specific antibodies targeting each isoform. QKI-5, QKI-6, QKI-7 each associated with miR-20a in doxorubicin-treated samples but not with pre-miR-20a or pri-miR20a. Ten percent of the Input cross-linked samples and $50 \%$ of the immunoprecipitates were analyzed by Western blotting for QKI (WB) to ensure similar immunoprecipitation of each QKI isoform. Each QKI isoform migrated in SDS-PAGE gel at their estimated sizes: QKI-5, $45 \mathrm{kDa}$; QKI-6, $38 \mathrm{kDa}$; QKI-7, $40 \mathrm{kDa} .\left(^{\star}\right) P<0.05$. (B) In vivo UV crosslinking using V5 antibody to immunoprecipitate V5-QKI-6 or V5-QKI-6 mutant from transiently transfected $293 \mathrm{~T}$ HEK as described in the Materials and Methods. Wildtype (WT) V5-QKI-6 associated with miR-20a but not pre-miR-20a or pri-miR-20a, while V5-QKI-6 mutant did not associate with any miRNA. $\left(^{\star \star}\right) P<0.01$. Ten percent of the Input cross-linked samples and $50 \%$ of the immunoprecipitates were analyzed by Western blotting with QKI antibody to ensure similar expression and immunoprecipitation of the in vivo cross-linked V5-QKI-6 and V5QKI-6 mutant proteins but not by the control Flag (Ctrl) antibody (the proteins' migration is affected due to the cross-linking incomplete nucleic acid removal). $(C)$ In vitro UV crosslinking was performed using purified V5-QKI6 and V5-QKI-6 mutant proteins with radio-labeled miRNAs, followed by immunoprecipitation with V5 agarose or control Flag agarose as described in the Materials and Methods. The immunoprecipitates were separated by SDS-PAGE, Western blotted, and analyzed by PhosphorImaging. Wild-type (WT) V5-QKI-6 purified protein associated with miR-20a but not with miR-18a, miR-20a ${ }^{\star}$, or pre-miR-20a; V5QKI-6 mutant purified proteins did not associate with any miRNA. To ensure similar immunoprecipitation of V5-QKI-6 and V5-QKI-6 mutant purified proteins and not with control Flag (Ctrl) antibody, these Western blots were reprobed with QKI antibody. (D) 293T HEK cells were transfected with vector, QKI-5, QKI-6, and QKI-7 all together (QKI) or mutant QKI-5, QKI-6, and QKI-7 all together (QKI-mut) for $24 \mathrm{~h}$ and then treated with $20 \mu \mathrm{g} / \mathrm{mL} \alpha$-amanitin. Cells were lysed every $3 \mathrm{~h}$ for up to $12 \mathrm{~h}$. QKI-transfected cells showed miR-20a stabilization relative to vector-transfected cells and QKI-mut-transfected cells. No difference in stabilization was observed for pre-miR-20a, pri-miR-20a, miR-18a, and miR-20a ${ }^{\star}$ mRNA. $\left(^{\star}\right) P<0.05$ compared with vector control. QKI expressions were displayed by Western blot.

(Fig. 3D). We did not observe QKI or QKI mutants affecting the stabilization of miR-18a, miR-20a ${ }^{\star}$, pre-miR-20a, or pri-miR-20a (Fig. 3D), suggesting that QKI specifically regulates mature miR-20a stability. Verifying this result, we also observed that QKI-5, QKI-6, and QKI-7 QKI isoforms each stabilized miR-20a but not miR-20a* (data not shown).

\section{miR-20a contributes to QKI-directed}

tumor suppression

We sought to document the tumor-suppressive role of the QKI-miR-20a axis specifically in human GBM cells, particularly in light of miR-20a's oncogenic activity in other tumor types (Ventura et al. 2008; Inomata et al. 2009).

To better understand the role of miR-20a in GBM pathogenesis, we first tested the ability for miR-20a to decrease soft agar colony formation, alongside its ability to reverse the increased soft agar colony formation produced by shRNA-mediated knockdown of QKI. When transiently transfecting synthetic miR-20a mimic and miRNA mimics of the other miRNAs in the miR-17-92 cluster into shQKI-Hs683 GBM cells (Supplemental Fig. 5A), only the miR-20a mimic blocked the increased colony formation generated by QKI knockdown (Supplemental Fig. 5B). 
Lentiviral transduction of miR-20a also decreased soft agar colony formation in shGFP cells and blocked the increased soft agar colony formation generated by QKI knockdown in both Hs683 (Fig. 4A) and U87 (Fig. 4B) human GBM cells. Furthermore, since the miR-17-92 cluster is oncogenic in colorectal cancers (Motoyama et al. 2009), we stably overexpressed miR-20a in the HCT116 colorectal cancer cell line as controls and observed increased soft agar colony formation, demonstrating context-specific roles for miR20a similar to other cancer genes, such as TGF $\beta$ (see the
Discussion) (Fig. 4C). Last, to validate in vivo, we lentivirally transduced miR-20a into shQki-Ink4a/Arf ${ }^{-1-}$ Pten $^{-/-}$ astrocytes, which led to a significant delay in the emergence of tumors relative to miR-NT-transduced shQki-Ink4a/ Arf $^{-1-}$ Pten $^{-/-}$astrocytes (Fig. 4D).

Next, we sought to delineate whether each QKI isoform regulates miR-20a. Hs683 cells transfected with siRNAs targeting each QKI-5, QKI-6, and QKI-7 isoform demonstrated that siRNA-mediated knockdown of specific QKI isoforms (Supplemental Fig. 5C) was associated
A.

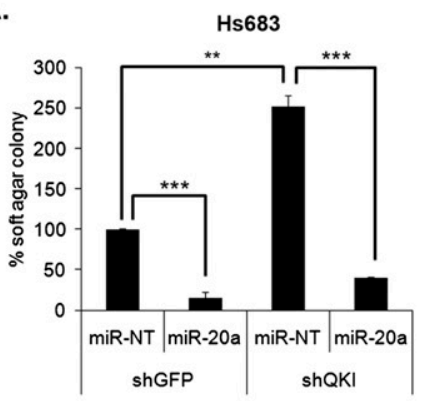

C.

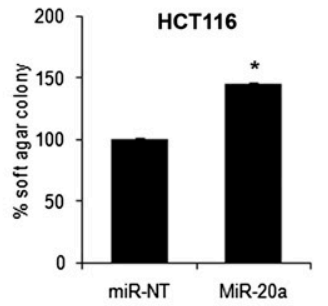

E.

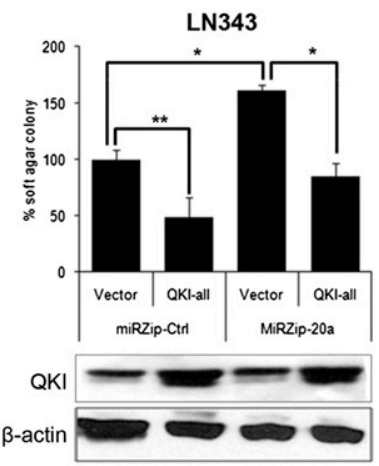

F.

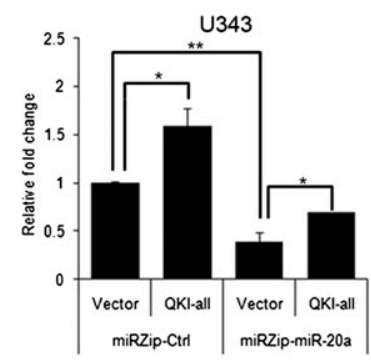

B.

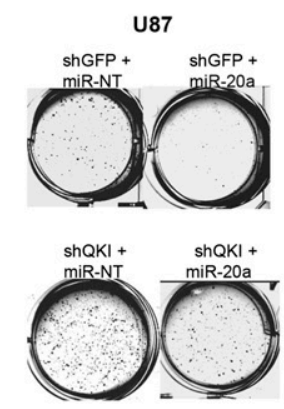

D.
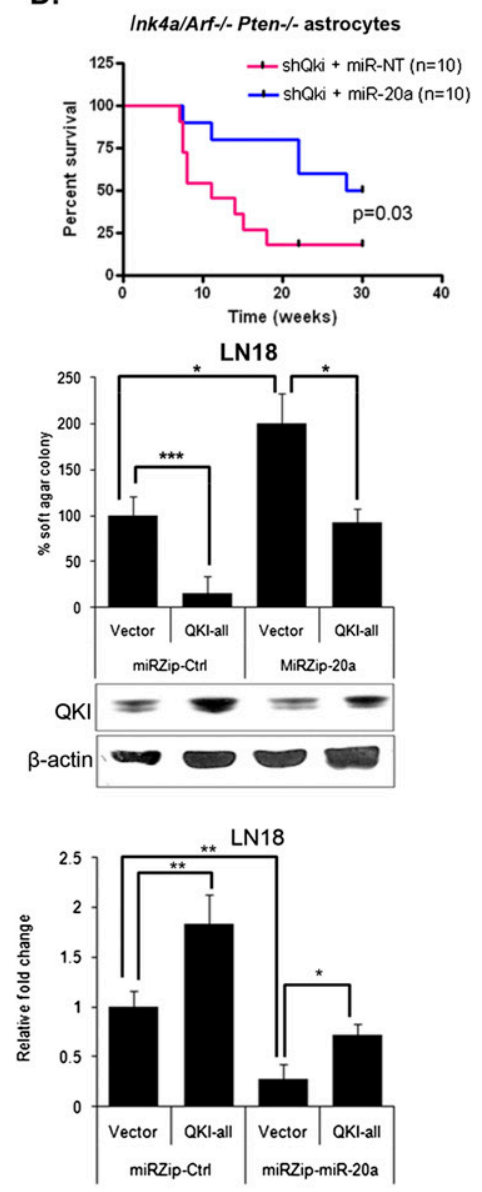

Figure 4. miR-20a partially carries out QKI tumor suppression. (A) Hs683-shGFP and Hs683-shQKI-2 were transiently transfected with miR-20a synthetic mimic or control mimic (miR-NT) and plated at 5000 cells per well in six-well plates for soft agar colony assay. Experiments were performed in duplicates. The miR-20a mimic decreased soft agar colony in shGFP cells relative to miR-NT control in shGFP cells. While shQKI and miR-NT double-transduced cells increased soft agar colony relative to shGFP and miR-NT double-transduced cells, miR-20a and shQKI doubletransduced cells decreased soft agar colony relative to shQKI and miR-NT double-transduced cells. Furthermore, miR-20a and shQKI double-transduced cells exhibited decreased colonies relative to miR-NT and shGFP double-transduced cells. $\left(^{\star \star}\right) P<0.01 ;\left(^{\star \star \star}\right) P<$ 0.001 compared with miR-NT. (B) U87-shGFP and U87-shQKI-2 were stably transduced with miR-20a or miR-NT and plated for soft agar colony assay. While miR-20a decreased soft agar colony in shGFP cells, miR-20a also decreased soft agar colony in shQKI cells relative to miR-NT controls. Magnification, $40 \times$. Experiments were performed in duplicates. $(C)$ HCT116 colorectal cancer cells were stably transduced with miR-20a or miR-NT, and 5000 cells per well were plated in six-well plates for soft agar colony assay. Experiments were performed in duplicates. miR-20a-transduced HCT116 showed increased soft agar colony formation relative to miR-NT-transduced cells. $\left.{ }^{*}\right) P<0.05$ compared with miR-NT. $(D)$ We injected $2 \times 10^{6}$ shQki-2-Ink4a/Arf ${ }^{-1-}$ Pten $^{-1-}$ astrocytes transduced with miR-20a or miR-NT subcutaneously into each flank of immuno-compromised nude mice, and Kaplan-Meier tumor-free survival curves were plotted. miR-20a-transduced shQki-2 cells showed significantly longer latencies in tumor formation relative to miR-NT-transduced shQki-2 cells $(P=0.03)$. $(E)$ All QKI isoforms were stably overexpressed in U343 and LN18 cells together with miRZip-Ctrl or miR-Zip-miR-20a for miR-20a knockdown; 5000 cells were plated per well in six-well plates for soft agar colony assay, and experiments were performed in duplicates. QKI overexpression decreased soft agar colony formation in miRZipCtrl-transduced U343 and LN18 cells. QKI overexpression also rescued the increased soft agar colony generated by miRZip-20a. $\left(^{\star}\right) P<0.05$; $\left.\left.\right|^{\star \star}\right) P<0.01$; $(\star \star \star) ~ P<0.001 .(F)$ miR-20a levels were measured by qPCR in U343 and LN18 transduced with vector or

QKI together with miRZip-Ctrl or miRZip-miR-20a for miR-20a knockdown. QKI overexpression increased miR-20a levels in miRZip-Ctrl-transduced U343 and LN18 cells, while QKI overexpression in miRZip-miR-20a cells rescued miR-20a expressions. $\left.\left(^{\star}\right) P<0.05 ;\left.\right|^{\star \star}\right) P<0.01$. 
with decreased miR-20a expression (Supplemental Fig. 5D). On the functional level, whereas soft agar colony formation increased in Hs683 and U87 cells transfected with siRNA against each QKI-5, QKI-6, and QKI-7 relative to the scrambled nontargeting siRNA control (siNT), these enhanced oncogenic effects of QKI isoform knockdown were reversed by enforced miR-20a expression (Supplemental Fig. 5E,F). Together, these functional studies support that miR-20a contributes to QKI-directed tumor suppressor actions in GBM.

To further verify the functional QKI-miR-20a link, we performed miR-20a knockdown by lentiviral stable inhibition (Supplemental Fig. 5G). miR-20a knockdown increased soft agar colony formation in Hs683 and U87 cells and in Ink4a/Arf ${ }^{-1-}$ Pten $^{-/-}$mouse astrocytes, but decreased soft agar colony formation in HCT116 cells (Supplemental Fig. $5 \mathrm{H})$. Furthermore, while miR-20a knockdown increased soft agar colonies in LN18 and U343 GBM cells, QKI overexpression rescued the miR-20a-mediated increase in soft agar colony formation (Fig. 4E). Consistent with our previous finding that QKI overexpression increased mature miR-20a levels (Supplemental Fig. 2F,G), QKI overexpression in LN18 and U343 partially rescued miR-20a levels in miR-20a knockdown cells (Fig. 4F).

\section{The QKI-miR-20a pathway partially inhibits TGFBR2} to suppress gliomagenesis

The above-noted QKI-directed regulation of the TGF $\beta$ pathway is noteworthy given previous studies demonstrating that TGF $\beta$ receptor 2 (TGF $\beta 22$ ) is a validated target of miR-20a (Volinia et al. 2006) and that TGFßR2 knockdown inhibits GBM cell tumor formation (Wesolowska et al. 2008). We therefore sought biochemical and functional evidence of a QKI-miR-20a-TGF $\beta$ R2 pathway in glioma systems. First, in Ink4a/Arf ${ }^{-1-}$ Pten $^{-1-}$ astrocytes, a progressive increase of Qki siRNA concentration produced a gradual decrease of miR-20a levels (see Supplemental Fig. 2C) and gradual increase of Tgf $\beta r 2$ protein levels (Fig. 5A). QKI knockdown (Supplemental Fig. 6A) or miR-20a knockdown (Supplemental Fig. 6B) did not affect TGF $\beta R 2$ mRNA levels, suggesting that miR-20a regulates TGF $\beta$ R2 mRNA via translational inhibition. Furthermore, QKI did not coimmunoprecipitate with TGFßR2 mRNA (Supplemental Fig. 6C), consistent with our finding that QKI inhibits TGF $\beta 2$ via miR-20a. Second, these TGF $\beta$ R2 changes corresponded well with downstream TGF $\beta$ pathway signaling in the context of QKI knockdown and miR20 a overexpression. Specifically, upon treatment with exogenous TGF $\beta 1$, U87 cells and Ink4a/Arf ${ }^{-1-}$ Pten $^{-/-}$ astrocytes showed shQKI-directed induction of TGF $\beta$ R2 and its downstream components, such as phosphoSMAD2 and p21 (Fig. 5B; Supplemental Fig. 6D). In these cell systems, enforced expression of miR-20a in shQKI cells reduced the expression of TGF $\beta R 2$ and its downstream effectors relative to miR-NT control in shQKI cells (Fig. 5B; Supplemental Fig. 6D), supporting the existence of the QKI-miR-20a-TGF $\beta$ pathway.

Next, to seek evidence of biological activity of this QKI-miR20a-TGF $\beta$ pathway, we performed rescue ex- periments in vitro. Briefly, as shown before (Fig. 1A,B), QKI knockdown resulted in increased soft agar colony formation in Hs683 and U87 cells (Fig. 5C,D). Correspondingly, knockdown of TGF $\beta 2$ by two independent shRNAs in this context reversed the anchorageindependent growth capability induced by shQKI (Fig. $5 \mathrm{C}, \mathrm{D})$. Significantly, knockdown of Tgf $\beta \mathrm{r} 2$ in vivo almost completely rescued the tumorigenic phenotype conferred by shQki in Ink4a/Arf ${ }^{-1-}$ Pten $^{-/-}$astrocytes (Fig. 5E).

Multidimensional genomic analysis of the TP53-QKI-miR-20a-TGFßR2 pathway in human GBM

We established here that (1) QKI is a novel p53 downstream target, and (2) QKI suppresses glioma development via a novel mechanism involving the stabilization of miR$20 \mathrm{a}$, which in turn inhibits TGF $\beta$ R2, a known GBM oncogene (Wesolowska et al. 2008). A model of the pathway is shown in Figure 6A. To obtain evidence for this model in human cancer genomes, we mined the multidimensional TCGA GBM data set for statistical evidence of an epistatic relationship across these genetic elements on the genomic and expression levels. First, as TP53 is frequently mutated in GBM (The Cancer Genome Atlas Research Network 2008; Zheng et al. 2008), we performed genomic analysis of the TCGA data sets on epistasis between TP53 mutation and QKI deletion. Specifically, while only $10 \%$ (nine out of 90 ) of the TP53 mutant cases harbor QKI deletion, 21.4\% (46 out of $215)$ of the TP53 wild-type tumors harbor $Q K I$ deletion $(P=$ 0.02 ) (Fig. 6B). Conversely, while $16.4 \%$ (nine out of 55 ) of the QKI-deleted patient samples carry TP53 mutation, 32.4\% (81 out of 250) of the QKI-nondeleted patients carry TP53 mutation $(P=0.02)$ (Fig. 6B). In other words, the genetic pressure for inactivating either TP53 or QKI is significantly reduced when either QKI or TP53 is already nonfunctional, respectively, supporting the functional relationship established by our data. Similarly, we performed expression correlation analyses to look for support of our model. In particular, as QKI stabilizes miR-20a, we expect their expressions to be positively correlated; correspondingly, as miR-20a inhibits TGFßR2, a negative correlation is expected. Indeed, we observed a significantly positive correlation of expression between QKI-miR-20a and a significantly negative correlation of expression between miR-20a-TGFßR2 among 389 samples in the TCGA data set (Fig. 6C; Supplemental Fig. 7). These significant correlations are notable because it is recognized that each of these components likely has additional activities and, conversely, is likely regulated by additional inputs. Therefore, the significant correlation of expressions suggests that the regulatory relationships in our model are sufficiently robust in human GBM.

In summary, both our proposed pathway and its mechanistic and functional interactions in glioma pathogenesis are strongly supported by the patterns of genomic and transcriptomic changes in the extensive human GBM patient sample in TCGA. While these data show strong interactions across these components, we emphasize that the actions of each component, including QKI, extend 
A.

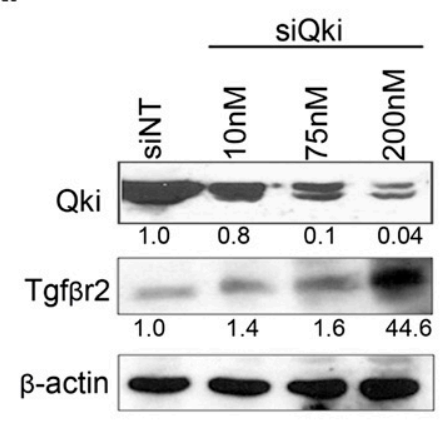

C.

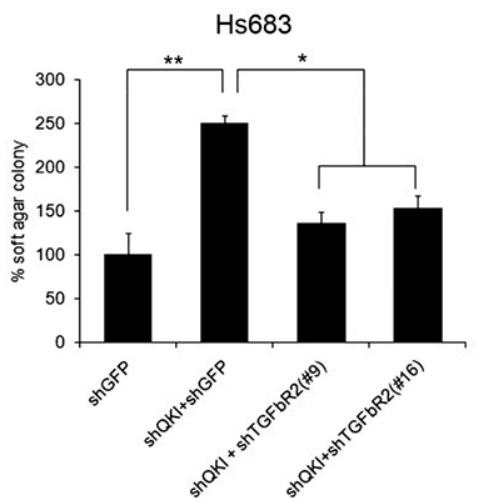

B. Ink4a/Arf-/- Pten-/- Astrocytes

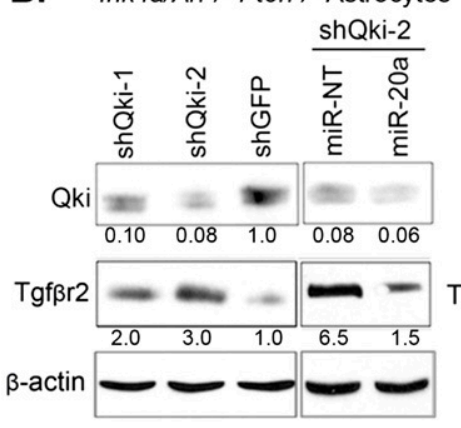

D.

U87

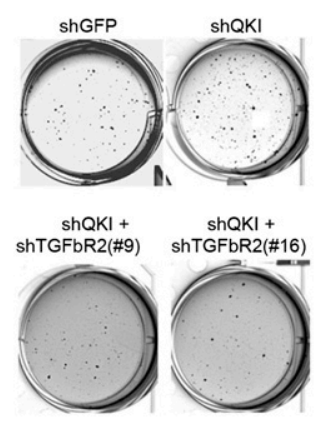

E.

Ink4a/Arf-/- Pten-/- Astrocytes

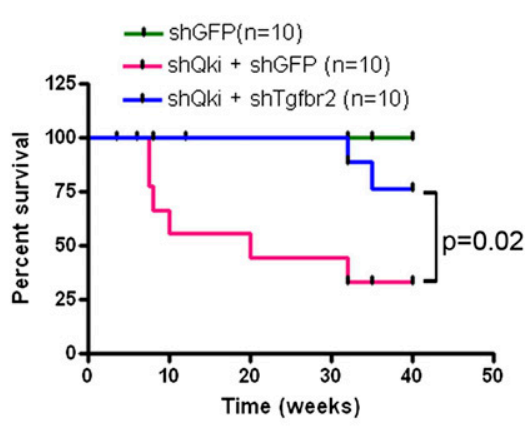

Figure 5. miR-20a carries out QKI tumor suppressor function partially via inhibiting TGF $\beta$ R2. (A) Increasing concentration of siRNA against Qki progressively up-regulated Tgf $\beta \mathrm{r} 2$ protein levels in Ink4a/Arf ${ }^{-1}$ Pten $^{-1}$ astrocytes. Relative protein levels for Qki and Tgf $\beta r 2$ were quantified in the Western blot. $(B)$ shQKI-2-transduced U87 and shQki-2-transduced Ink4a/Arf ${ }^{-1-}$ Pten $^{-/-}$astrocytes exhibited elevated TGF $\beta$ R2 protein levels, as quantified by Western blot. Stable overexpression of miR-20a reversed the increased TGF $\beta R 2$ protein levels in both cell types. (C) Hs683 with QKI knockdown increased soft agar colony formation. TGF $\beta$ R2 knockdown by two independent shRNAs rescued the increased soft agar colony formation generated by QKI knockdown. Five-thousand cells were plated per well in six-well plates, and experiments were performed in duplicates. $\left.\left(^{\star}\right) P<0.05 ;\left.\right|^{\star \star}\right) p<0.01$. $(D)$ U87 transduced with shQKI increased soft agar colony formation. TGF $\beta$ R 2 knockdown by two independent shRNAs rescued the increased soft agar colony formation generated by QKI knockdown. Magnification, 40×. Five-thousand cells were plated per well in six-well plates, and experiments were performed in duplicates. (E) We injected $2 \times 10^{6}$ shQki-2-Ink4a/Arf ${ }^{-1-}$ Pten ${ }^{-1-}$ mouse astrocytes transduced with shRNA against Tgfßr2 or GFP subcutaneously into each flank of immuno-compromised nude mice, and Kaplan-Meier tumor-free survival curves were plotted. While shQki-transduced cells formed tumors, shGFP-transduced cells did not form tumors. ShTgf $\beta r 2-$ transduced cells rescued the tumor formation enhanced by shQki-transduced cells $(P=0.02)$.

beyond this linear pathway to include additional targets and cancer pathways, as shown in Figure 6A.

\section{Discussion}

In this study, we validated QKI as a downstream target of p53 that functions to suppress tumorigenesis via stabilization of miR-20a, which in turn inhibits TGFßR2 and its downstream targets. These data establish a new tumor suppressor pathway of gliomagenesis and reveal a novel mechanism of tumor suppression involving QKImediated stabilization of a cancer-relevant miRNA. This p53-QKI-miR-20a-TGFßR2 pathway circuitry was validated on the molecular, biochemical, and functional levels in vitro and in vivo using both mouse primary astrocytes and human transformed glial cell systems. In addition, the proposed pathway aligns with the profiles of human GBM based on TCGA data, wherein sufficient sample size and multidimensionality provided robust statistical support for the genetic interactions between each connection in this pathway.

The identification of QKI as a p53-regulated GBM tumor suppressor gene gains significance in light of recent genetic and genomic data that establish potent cooperative tumor suppressor activity between $p 53$ and Pten in mice (Zheng et al. 2008) and documents TP53 as the most highly mutated gene in human GBM (The Cancer Genome Atlas Research Network 2008). These observations add another physiologically relevant dimension to p53's web of tumor suppressor activities that have recently been expanded to include the regulation of noncoding RNAs, such as miRNA transcriptional activation (He et al. 2007a) and processing (Suzuki et al. 2009). Our study now provides evidence that p53 can regulate the 
A.

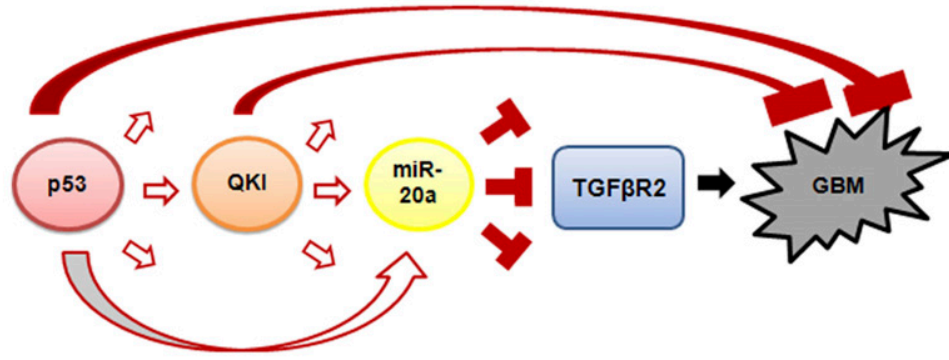

B.

\begin{tabular}{|l|c|c|c|}
\hline & TP53 mutation & TP53WT & p-value \\
\hline QKI deletion & $9 / 90(10 \%)$ & $46 / 215(21.4 \%)$ & $\mathrm{p}=0.02$ \\
\hline & QKIdeletion & QKInon-deletion & \\
\hline & $9 / 55(16.4 \%)$ & $81 / 250(32.4 \%)$ & $\mathrm{p}=0.02$ \\
\hline TP53 mutation &
\end{tabular}

C.

\begin{tabular}{|c|c|c|c|c|}
\hline species1 & species 2 & $\begin{array}{c}\text { expected } \\
\text { correlation }\end{array}$ & correlation & p-value \\
\hline QKI & miR-20a & + & 0.149 & 0.005 \\
\hline miR-20a & TGFbR2 & - & -0.28 & $<0.0001$ \\
\hline
\end{tabular}

Figure 6. QKI mechanism correlates with expression and genomic alterations in TCGA human GBM patient samples. (A) A model for the mechanism of QKI in GBM tumor suppression. While p53 can transcriptionally activate both QKI and miR-20a, QKI has an independent role of upregulating miR-20a at least partially through stabilization. miR-20a in turn inhibits TGF $\beta$ R2, an oncogene in gliomagenesis. p53, QKI, and miR20a can additionally act on downstream targets other than those proposed in this model. (B) TP53 and $Q K I$ demonstrate epistatic relationships in TCGA genomic analysis. $(C)$ An analysis for transcript expression correlation among 389 human GBM patients in TCGA. QKI is positively correlated with miR-20a. miR-20a is negatively correlated with TGF $\beta$ R2. transcriptional activation of RNA-binding protein QKI to increase levels of a glioma-suppressive miRNA via stabilization, thereby connecting two major glioma-relevant cancer networks: p53 and TGF $\beta$. Nevertheless, QKI is in the same minimal common region on chromosome 6q2627 as the recently published GBM tumor suppressor gene PARK2 (Veeriah et al. 2010), and our study showed that PARK2 and QKI confer an additive effect in tumor suppression. Thus, it is possible that dual inactivation of $P A R K 2$ and QKI act cooperatively in driving GBM, although focal $Q K I$ deletions also underscore its pathogenetic relevance in this disease.

The role of RNA-binding proteins in the pathogenesis of cancer rests on strong experimental support (Busa et al. 2007; Richard et al. 2008; Guo et al. 2009). As RNA-binding proteins can partake in all aspects of posttranscriptional regulation, they carry significant implications in understanding cancer pathogenesis and its treatment. Along these lines, the RNA-binding protein $\mathrm{HuR}$ is often overexpressed in breast cancer /Guo et al. 2009), while Sam68 is a known oncogene in breast and prostate cancers (Busa et al. 2007; Richard et al. 2008). Together with our finding that QKI is a GBM tumor suppressor gene that exerts its tumor-suppressive effect at least in part through the stabilization of a specific miRNA, miR-20a, these collective observations establish the growing general importance of RNA metabolism in cancer pathogenesis. Our study also establishes that the RNA-binding protein QKI associates with miR-20a to stabilize and maintain this specific miRNA's levels and exert a tumor-suppressive effect in GBM. Interestingly, QKI associates with the mature miR-20a but not other
miRNAs in the same cluster and leads to stabilization of only the miR-20a guide strand but not the miR-20a* passenger strand. Importantly, the $\mathrm{KH}$ domain of QKI is essential for miR-20a interaction and stabilization (Fig. 3), consistent with a previous study demonstrating a $\mathrm{KH}$ domain requirement for direct interaction of the RNAbinding protein hnRNP E2 with another specific miRNA, miR328 (Eiring et al. 2010). How QKI and other KH domain proteins like hnRNP E2 achieve such selectivity will require further study. While a previous study showed that QKI interacts with Argonautes in the RISC complex (Wang et al. 2010), our study reveals that QKI also has direct miRNA regulatory functions. At the same time, since QKI is known to regulate many aspects of mRNA metabolism, including alternative splicing (Wu et al. 2002) and transport (Larocque et al. 2002), we acknowledge that QKI may regulate additional cancer targets and pathways via additional mechanisms in a manner similar to virtually all cancer genes.

The role of TGF $\beta$ signaling in cancer is context-driven and can be either tumor-suppressive or oncogenic, depending on cancer type, stage of tumor formation (e.g., initiation or progression), and cross-talk with other cytokines and signaling factors (Rich 2003; Hjelmeland et al. 2004). As a regulator of TGF $\beta$ R2 expression and thus TGF $\beta$ signaling, the role of miR-20a is also tissue-specific. While our study showed that miR-20a is tumor-suppressive and TGF $\beta R 2$ is oncogenic in GBM, previous studies have shown miR-20a as oncogenic (Ota et al. 2004; Hayashita et al. 2005; Ventura et al. 2008; Inomata et al. 2009; Motoyama et al. 2009) and TGF $\beta$ signaling as tumor-suppressive (Dews et al. 2010) in other tumor types; for instance, miR-20a is oncogenic and 
TGFßR2 is tumor-suppressive in colon cancer (Dews et al. 2010). These tissue-specific differences are further exemplified by the fact that p53 transcriptionally suppresses the miR-17-92 cluster in colon cancer (Yan et al. 2009), whereas p53 transcriptionally activates the miR-17-92 cluster in GBM (this study). Interestingly, although miR-20a and miR17 share seed sequence, miR-17 did not rescue the increased tumorigenicity generated by QKI knockdown, as miR-20a did. This observation points to different functions and/or modes of regulation of miR-17 and miR-20a in GBM, as previously suggested (Ernst et al. 2010; Malzkorn et al. 2010).

The complexity of TGF $\beta$ signaling makes drug development targeting either TGF $\beta$ or the TGF $\beta$ receptors especially demanding, since these therapies must be applied only at selective types and stages of cancers. Enabled by TCGA in novel cancer gene discovery and in silico analysis of genetic interactions, we can now begin to address such challenging clinical issues. For instance, as we were able to dissect the TP53-QKI-miR-20a-TGFßR2 pathway and validate the association of each component through expression and genomic data of the TCGA data set, it is tempting to speculate that QKI status could serve as a biomarker for TGF $\beta$ inhibitor therapies in GBM. As our understanding of cancer pathogenesis advances, the TCGA effort will ultimately provide more granularity on pathway circuitry, leading to more informed therapeutic responses and targeted therapy for this dreaded disease.

\section{Materials and methods}

\section{Cell culture}

The human glioma cell lines U343, LN18, U87, and Hs683 and the cell line HEK293T were purchased from American Type Culture Collection (ATCC). Primary murine astrocytes were isolated from 5-d-old pups with indicated genotypes and maintained in DMEM containing 10\% FBS as previously described (Bachoo et al. 2002).

\section{Plasmids, shRNA, and siRNA}

QKI-5, QKI-6, and QKI-7 were amplified from a mouse embryonic cDNA library using the following primers: forward primer, ATGGTCGGGGAAATGGAAACGAAGGAGAAGCCG; QKI-5 reverse primer, TTAAGCGTAGTCTGGGACTGCTGATCCGT ACCAGTTAAC; QKI-6 reverse primer, TTAAGCGTAGTCT GGGACGTCGTATGGGTAGCCTTTCGTTGGGAAAGC; and QKI-7 reverse primer, TCAAGCGTAGTCTGGGACGTCGTAT GGGTAATGGGCTGAAATA. QKI-5, QKI-6, and QKI-7 were cloned into the pLenti6-V5 vector (Invitrogen) for overexpression using the Gateway System. V5-QKI-6 was cloned into the pLenti6-V5 vector with the reverse primer without the stop codon, AGCGTAGTCTGGGACGTCGTATGGGTAGCCTTTCG TTGGGAAAGC.

shRNAs against QKI were received from the RNAi Consortium of the Broad Institute. shRNA target sequences are the following: shQKI-1, GACGAAGAAATTAGCAGAGTA (TRCN0000015187); shQKI-2, CCCTACCATAATGCCTTTG AT (TRCN0000015184); shQki-1, GCCTTCAGATAGTTGAGA ATA (TRCN0000102405); shQki-2, CCTAGAGGACTTACAG CTAAA (TRCN0000102406); shTGFBR2 \#9, GCAGAACACTT CAGAGCAGTT (TRCN0000040011); shTGFBR2 \#16, CAA
CAACGGTGCAGTCAAGTT (TRCN0000010444); shTgfbr2, CGACCTGTTGTTGGTCATTAT (TRCN0000022624); shPARK2, CTTAGACTGTTTCCACTTATA (TRCN0000000285); and shPark2, GACCTGGAACAACAGAGTATT (TRCN0000041147).

siRNAs against QKI isoforms were custom-made and ordered from Dharmacon. Target sequences are the following: siQKI-5, CUGGUAAUCGCCUUUGCUU; siQKI-6, AGUGGUGUAUU AGGUAUGG; and siQKI-7, GAGUGGAUUGAAAUGCCA.

\section{ChIP analysis}

For ChIP, we followed the protocol provided with the EZ ChIP kit from Upstate Biotechnology. In short, normal human astrocytes from five $15-\mathrm{cm}$ plates were fixed by paraformaldehyde, lysed, and sheared by sonication (three times for $10 \mathrm{sec}$, idle time $45 \mathrm{sec}$. For overnight immunoprecipitations, $5 \mu \mathrm{g}$ of a mouse p53 antibody (DO-1, Santa Cruz Biotechnology, sc-126) or an equal amount of control mouse IgG (Bethyl Laboratories, MI10102) was used. After extensive washing, the immunoprecipitants were eluted with $2 \% \mathrm{SDS}$ in $0.1 \mathrm{M} \mathrm{NaH}_{2} \mathrm{CO}_{3}$. Cross-linking was reversed by heating overnight at $65^{\circ} \mathrm{C}$, and samples were treated with proteinase $\mathrm{K}$ for $1 \mathrm{~h}$ at $45^{\circ} \mathrm{C}$. Input DNA and immunoprecipitated DNA were purified using the PCR purification kit (Qiagen) and analyzed by PCR and qPCR. PCR products were subsequently separated on $2 \%$ agarose gels to visualize.

\section{Adenoviral p53 analysis}

For adenoviral delivery of p53, Ad-p53-GFP was commercially purchased (Vector Laboratories, \#1260) and $p 53^{-/-}$primary mouse astrocytes were infected at $80 \mathrm{MOI}$ (multiplicity of infection). Cells were harvested $36 \mathrm{~h}$ after infection and processed for RNA analysis.

\section{Mutagenesis}

QKI mutagenesis was performed using the QuikChange II sitedirected mutagenesis kit (Stratagene) following the manufacturer's instructions. The primers used were CGGCTGCT GGACGAAGGAATTAGCAGAGTACGG (QKI-a143g) and CC GTACTCTGCTAATTCCTTCGTCCAGCAGCCG (QKI-a143g antisense).

\section{Microarray expression analysis}

RNA was isolated using TRIzol (Invitrogen) and the RNeasy minikit (Qiagen). Gene expression profiling was performed using the Affymetrix Human Genome U133 Plus 2.0 array for human GBM cells and the Affymetrix Mouse Genome 4302.0 array for primary Ink $4 a / \mathrm{Arf}^{-1-}$ Pten $^{-1-}$ primary mouse astrocytes. Profiling was performed at the DFCI Microarray Core Facility.

\section{miRNA isolation, $R T$, and $q P C R$}

Total RNA was purified using the High Pure miRNA Isolation kit (Roche) and reverse-transcribed using the miScript RT kit (Qiagen) following the manufacturer's instructions. qPCR was performed using the miScript SYBR Green PCR kit (Qiagen). Results are presented as percent input following the formula $100 \times 2^{\text {(Adjusted input }-\mathrm{Ct}(\text { IP) }}$.

\section{miRNA overexpression and knockdown}

Transient transfection of the synthetic miR-20a mimic was performed using miScript miRNA mimics (Qiagen). Lentiviral transduction for stable overexpression of miR-20a or scrambled 
miRNA was performed by using miExpress Precursor miRNA Expression Clones (GeneCopoeia). Lentiviral transduction for stable knockdown of miR-20a or a control miRNA was performed by using the miRZips System (System Biosciences).

\section{Protein-RNA immunoprecipitation analysis}

Polysome lysis buffer was prepared as previously described (Keene et al. 2006). Briefly, $100 \mathrm{mM} \mathrm{KCl}, 5 \mathrm{mM} \mathrm{MgCl}_{2}, 10 \mathrm{mM}$ HEPES (pH 7.0), and 0.5\% NP-40 were made in RNase-DNasefree $\mathrm{H}_{2} \mathrm{O}$. One millimolar DTT, $100 \mathrm{U} / \mathrm{mL}$ RNase Out (Promega), $400 \mu \mathrm{M}$ VRC, and Protease inhibitor cocktail (Roche Complete Mini) were added at the time of use. Protein-RNA immunoprecipitation was performed using the Seize $\mathrm{X}$ immunoprecipitation kit (Pierce). The antibodies used were QKI (A300-182A, Bethyl Laboratories) and Sc35 (S4045-0.2ml, Sigma).

\section{Immunoblotting}

Cells were lysed in RIPA buffer supplemented with protease inhibitor (Roche Complete minitablet) and phosphatase inhibitor (Pierce Halt phosphatase inhibitor cocktail). Protein lysates were run on a NuPAGE Bis-Tris $4 \%-12 \%$ gradient gel (Invitrogen) and transferred onto a PVDF membrane (Immobilon P, Millipore). Detection was performed with an enhanced chemiluminescence (ECL) system (SuperSignal West Pico, Thermo Scientific).

The antibodies used for Western blots were $\beta$-actin (AC-74, Sigma), QKI (A300-182A, Bethyl Laboratories), p21 (sc-397, Santa Cruz Biotechnology), p53 (sc-126, Santa Cruz Biotechnology; CM5, Vector Laboratories), HA (12CA5, Roche), Smad2 (\#3122, Cell Signaling), pSmad2 (\#3101, Cell Signaling), and TGFßR2 (sc400, Santa Cruz Biotechnology).

\section{Protein purification}

V5-tagged QKI-6 and V5-tagged QKI-6 mutant were transiently transfected individually into 293T HEK cells. Protein purification was performed using the V5-tagged Protein Purification kit (catalog no. 3315, MBL). Purified proteins were run on a NuPAGE Bis-Tris $4 \%-12 \%$ gradient gel (Invitrogen) and subjected to Coomassie Blue incubation.

\section{Radioactive-labeled RNA}

RNA was labeled using a T7 MegaScript kit (AM1338, Ambion) using $\left[\alpha-{ }^{32} \mathrm{P}\right] \mathrm{dUTP}$ isotopes. The primers used were T7-miR-20a (forward, GTAATACGACTCACTATAGGGTACTCAAGCTAA AGTGCTTATAGTGCAGGTAG; reverse, CTACCTGCACTA TAAGCACTTTAGCTTGAGTACCCTATAGTGAGTCGTAT TAC), T7-miR-20a* (forward, GTAATACGACTCACTATAG GGTACTCAAGCACTGCATTATGAGCACTTAAAG; reverse, CTTTAAGTGCTCATAATGCAGTGCTTGAGTACCCTATAG TGAGTCGTATTAC), T7-miR-18a (forward, GTAATACGACTC ACTATAGGGTACTCAAGCTAAGGTGCATCTAGTGCAGA TAG; reverse, CTATCTGCACTAGATGCACCTTAGCTTGAG TACCCTATAGTGAGTCGTATTAC), and T7-pre-miR-20a (forward, GTAATACGACTCACTATAGGGTACTCAAGCGTAGCA CTAAAGTGCTTATAGTGCAGGTAGTGTTTAGTTATCTACT GCATTATGAGCACTTAAAGTACTGC; reverse, GCAGTACTT TAAGTGCTCATAATGCAGTAGATAACTAAACACTACCTG CACTATAAGCACTTTAGTGCTACGCTTGAGTACCCTATA GTGAGTCGTATTAC). The labeled RNAs were either gelpurified by $16 \%$ urea PAGE and/or G-25 column-purified followed by phenol chloroform extraction twice and ethanol precipitation.
In vitro $U V$ cross-linking

Cell lysates or $200 \mathrm{nM}$ purified proteins (V5-QKI-6 or V5-QKI-6 mutant) together with T7-transcribed, $\mathrm{P}^{32}$-UTP-labeled small RNAs in binding buffer $(10 \mathrm{nM}$ RNA, $5 \%$ glycerol, $0.1 \mu \mathrm{g} / \mu \mathrm{L}$ BSA, 2\% PVA, $1.5 \mathrm{mM} \mathrm{MgCl}_{2}, 5 \mathrm{mM} \mathrm{DTT}$ ) incubated for $10 \mathrm{~min}$ at room temperature were cross-linked using short-wavelength UV light $(254 \mathrm{~nm})$ for $10 \mathrm{~min}$ on ice. Samples were then treated with RNase A/T1 for $10 \mathrm{~min}$ at room temperature. Samples were then incubated with V5 beads in binding buffer $150 \mathrm{mM} \mathrm{NaCl}$, $40 \mathrm{mM}$ HEPES 7.0, $6 \mathrm{mM} \mathrm{MgCl}_{2}, 0.05 \%$ NP-40, 1 mM DTT, $10 \%$ glycerol, $1 \mathrm{mM}$ PMSF) overnight, washed three times with RIPA buffer ( $1 \%$ NP-40, 0.5\% sodium deoxycholate, 0.05\% SDS, 150 $\mathrm{mM} \mathrm{NaCl}, 50 \mathrm{mM}$ Tris 7.0), and then subjected to SDS-PAGE and Western blotted onto nitrocellulose. The cross-linked proteins are radiolabeled by the labeled miRNAs bound and were detected using a PhosphorImager. The blots were then reprobed to ensure equal immunoprecipitation of V5-QKI-6 and V5-QKI-6 mutant.

\section{In vivo UV cross-linking}

293T HEK cells were transiently transfected with V5-QKI-6 and V5-QKI-6 mutant. UV cross-linking was performed at $3400 \mu \mathrm{J} / \mathrm{cm}^{2}$ with the cells covered in a layer of $12 \mathrm{~mL}$ of PBS buffer in a $15-\mathrm{cm}$ diameter plate using the Stratalinker (Stratagene) (Ule et al. 2003). Cells were scraped off the plates, washed with PBS and sonicated four time for $5 \mathrm{sec}$ with $30 \mathrm{sec}$ of cooling on ice in $500 \mu \mathrm{L}$ of cold lysis buffer (150 mM NaCl, $40 \mathrm{mM}$ HEPES 7.0, $6 \mathrm{mM} \mathrm{MgCl}_{2}$, $0.1 \%$ NP-40, 1 mM DTT, $10 \%$ glycerol, $1 \%$ PMSF), and the extracts were clarified at $1000 \mathrm{rpm}$ for $10 \mathrm{~min}$. The extracts were subjected to V5 immunoprecipitation using preblocked V5 beads (preblocked with $1 \mathrm{mg} / \mathrm{mL}$ glycogen in binding buffer without NP-40 or DTT for $1 \mathrm{~h}$ followed by clarification of the beads to remove the excess buffer prior to adding the extracts) in the lysis buffer with $0.5 \mu \mathrm{L}$ of RNase inhibitor overnight, following which the beads were washed three times with RIPA buffer and once with binding buffer. Fifty percent of the washed immunoprecipitates were treated with proteinase $\mathrm{K}(30 \mathrm{mg} / \mathrm{mL})$ for $15 \mathrm{~min}$ at $37^{\circ} \mathrm{C}$ the next day prior to RNA isolation and qPCR analyses, while the rest of the immunoprecipitates were treated with Micrococcal Nuclease and RNase One prior to acetone precipitation, followed by resuspension in $2 \times$ SDS-PAGE loading buffer with $0.2 \mathrm{M}$ DTT, SDS-PAGE separation, and Western analyses for QKI.

\section{Genomic and statistical analysis}

Raw data from the TCGA Agilent 244K array-CGH platform (Harvard Center, 339 samples) were normalized using loess normalization for dye bias correction and segmented by the Circular Binary Segmentation algorithm (Lai et al. 2005). The CNTools package (http://www.bioconductor.org/packages/2.5/bioc/html/ CNTools.html) was used to assign copy number change ( $\log _{2}$ ratio) values to all genes across the samples. Genes were designated to be in regions of copy number gain or loss based on threshold values of $>0.4$ and less than -0.4 , respectively. Normalized TCGA expression data (level 3) for 290 samples from the University of North Carolina and mutation data from Washington University (St. Louis), Broad Institute, and Baylor College of Medicine were used in the analysis (Venkatraman and Olshen 2007; The Cancer Genome Atlas Research Network 2008). The first and third quartiles of the expression distribution were used to define samples with underexpression or overexpression of a particular gene.

Survival curves were generated using the Kaplan-Meier method, and the statistical significance of the difference between survival 
curves was assessed by the log-rank test. The significance of calculated Pearson correlations was computed using the $t$-statistic

$$
t_{n-2}=r \sqrt{\frac{n-2}{1-r^{2}}}
$$

where $r$ is the correlation, and $n$ is the number of samples.

\section{Acknowledgments}

We thank H. Zheng, J. Stommel, E. Sahin, and F. Muller, as well as the members of the DePinho, Chin, and Vasudevan laboratories for advice and support. J.H.P. was supported by the Damon Runyon Cancer Research Foundation. J.H. was supported by a Leukemia and Lymphoma Society Fellowship. Grant support comes from the Goldhirsh Foundation (to R.A.D.) and NIH grants RO1CA99041 (to L.C.), 5P01CA95616 (to L.C. and R.A.D.), and GM057476 (to P.A.S.). R.A.D. is an American Cancer Society Research Professor supported by the Robert A. and Renee E. Belfer Foundation Institute for Innovative Cancer Science.

\section{References}

Aberg K, Saetre P, Jareborg N, Jazin E. 2006. Human QKI, a potential regulator of mRNA expression of human oligodendrocyte-related genes involved in schizophrenia. Proc Natl Acad Sci 103: 7482-7487.

Bachoo RM, Maher EA, Ligon KL, Sharpless NE, Chan SS, You MJ, Tang Y, DeFrances J, Stover E, Weissleder R, et al. 2002. Epidermal growth factor receptor and Ink4a/Arf: Convergent mechanisms governing terminal differentiation and transformation along the neural stem cell to astrocyte axis. Cancer Cell 1: 269-277.

Busa R, Paronetto MP, Farini D, Pierantozzi E, Botti F, Angelini DF, Attisani F, Vespasiani G, Sette C. 2007. The RNA-binding protein Sam68 contributes to proliferation and survival of human prostate cancer cells. Oncogene 26: 4372-4382.

Calin GA, Dumitru CD, Shimizu M, Bichi R, Zupo S, Noch E, Aldler H, Rattan S, Keating M, Rai K, et al. 2002. Frequent deletions and down-regulation of micro-RNA genes miR15 and miR16 at 13q14 in chronic lymphocytic leukemia. Proc Natl Acad Sci 99: 15524-15529.

The Cancer Genome Atlas Research Network. 2008. Comprehensive genomic characterization defines human glioblastoma genes and core pathways. Nature 455: 1061-1068.

Chen T, Richard S. 1998. Structure-function analysis of Qk1: A lethal point mutation in mouse quaking prevents homodimerization. Mol Cell Biol 18: 4863-4871.

Christophorou MA, Martin-Zanca D, Soucek L, Lawlor ER, Brown-Swigart L, Verschuren EW, Evan GI. 2005. Temporal dissection of p53 function in vitro and in vivo. Nat Genet 37: 718-726.

Dews M, Homayouni A, Yu D, Murphy D, Sevignani C, Wentzel E, Furth EE, Lee WM, Enders GH, Mendell JT, et al. 2006. Augmentation of tumor angiogenesis by a Myc-activated microRNA cluster. Nat Genet 38: 1060-1065.

Dews M, Fox JL, Hultine S, Sundaram P, Wang W, Liu YY, Furth E, Enders GH, El-Deiry W, Schelter JM, et al. 2010. The mycmiR-17 92 axis blunts TGF $\beta$ signaling and production of multiple TGF $\beta$-dependent antiangiogenic factors. Cancer Res 70: 8233-8246.

Ebersole TA, Chen Q, Justice MJ, Artzt K. 1996. The quaking gene product necessary in embryogenesis and myelination combines features of RNA binding and signal transduction proteins. Nat Genet 12: 260-265.
Eiring AM, Harb JG, Neviani P, Garton C, Oaks JJ, Spizzo R, Liu S, Schwind S, Santhanam R, Hickey CJ, et al. 2010. miR-328 functions as an RNA decoy to modulate hnRNP E2 regulation of mRNA translation in leukemic blasts. Cell 140: 652-665.

Ernst A, Campos B, Meier J, Devens F, Liesenberg F, Wolter M, Reifenberger G, Herold-Mende C, Lichter P, Radlwimmer B. 2010. De-repression of CTGF via the miR-17-92 cluster upon differentiation of human glioblastoma spheroid cultures. Oncogene 29: 3411-3422.

Esquela-Kerscher A, Slack FJ. 2006. Oncomirs-microRNAs with a role in cancer. Nat Rev Cancer 6: 259-269.

Guo X, Wu Y, Hartley RS. 2009. MicroRNA-125a represses cell growth by targeting HuR in breast cancer. RNA Biol 6: 575-583.

Haroutunian V, Katsel P, Dracheva S, Davis KL. 2006. The human homolog of the QKI gene affected in the severe dysmyelination 'quaking' mouse phenotype: Downregulated in multiple brain regions in schizophrenia. Am I Psychiatry 163: 1834-1837.

Hayashita Y, Osada H, Tatematsu Y, Yamada H, Yanagisawa K, Tomida S, Yatabe Y, Kawahara K, Sekido Y, Takahashi T. 2005. A polycistronic microRNA cluster, miR-17-92, is overexpressed in human lung cancers and enhances cell proliferation. Cancer Res 65: 9628-9632.

He L, He X, Lim LP, de Stanchina E, Xuan Z, Liang Y, Xue W, Zender L, Magnus J, Ridzon D, et al. 2007a. A microRNA component of the p53 tumour suppressor network. Nature 447: 1130-1134.

He L, He X, Lowe SW, Hannon GJ. 2007b. microRNAs join the p53 network-another piece in the tumour-suppression puzzle. Nat Rev Cancer 7: 819-822.

Hjelmeland MD, Hjelmeland AB, Sathornsumetee S, Reese ED, Herbstreith $\mathrm{MH}$, Laping NJ, Friedman HS, Bigner DD, Wang XF, Rich JN. 2004. SB-431542, a small molecule transforming growth factor- $\beta$-receptor antagonist, inhibits human glioma cell line proliferation and motility. Mol Cancer Ther 3: 737-745.

Inomata $\mathrm{M}$, Tagawa $\mathrm{H}$, Guo YM, Kameoka $\mathrm{Y}$, Takahashi N, Sawada K. 2009. MicroRNA-17-92 down-regulates expression of distinct targets in different B-cell lymphoma subtypes. Blood 113: 396-402.

Kataoka N, Fujita M, Ohno M. 2009. Functional association of the Microprocessor complex with the spliceosome. Mol Cell Biol 29: 3243-3254.

Keene JD, Komisarow JM, Friedersdorf MB. 2006. RIP-Chip: The isolation and identification of mRNAs, microRNAs and protein components of ribonucleoprotein complexes from cell extracts. Nat Protoc 1: 302-307.

Lai WR, Johnson MD, Kucherlapati R, Park PJ. 2005. Comparative analysis of algorithms for identifying amplifications and deletions in array CGH data. Bioinformatics 21: 3763 3770.

Larocque D, Pilotte J, Chen T, Cloutier F, Massie B, Pedraza L, Couture R, Lasko P, Almazan G, Richard S. 2002. Nuclear retention of MBP mRNAs in the quaking viable mice. Neuron 36: 815-829.

Larocque D, Galarneau A, Liu HN, Scott M, Almazan G, Richard S. 2005. Protection of p27(Kip1) mRNA by quaking RNA binding proteins promotes oligodendrocyte differentiation. Nat Neurosci 8: 27-33.

Li ZZ, Kondo T, Murata T, Ebersole TA, Nishi T, Tada K, Ushio Y, Yamamura K, Abe K. 2002. Expression of Hqk encoding a KH RNA binding protein is altered in human glioma. Ipn $J$ Cancer Res 93: 167-177.

Li Z, Takakura N, Oike Y, Imanaka T, Araki K, Suda T, Kaname T, Kondo T, Abe K, Yamamura K. 2003. Defective smooth 
muscle development in qkI-deficient mice. Dev Growth Differ 45: 449-462.

Lobbardi R, Lambert G, Zhao J, Geisler R, Kim HR, Rosa FM. 2011. Fine-tuning of Hh signaling by the RNA-binding protein Quaking to control muscle development. Development 138: $1783-1794$.

Maher EA, Brennan C, Wen PY, Durso L, Ligon KL, Richardson A, Khatry D, Feng B, Sinha R, Louis DN, et al. 2006. Marked genomic differences characterize primary and secondary glioblastoma subtypes and identify two distinct molecular and clinical secondary glioblastoma entities. Cancer Res 66: 11502-11513.

Malzkorn B, Wolter M, Liesenberg F, Grzendowski M, Stuhler K, Meyer HE, Reifenberger G. 2010. Identification and functional characterization of microRNAs involved in the malignant progression of gliomas. Brain Pathol 20: 539550.

Maser RS, Choudhury B, Campbell PJ, Feng B, Wong KK, Protopopov A, O'Neil J, Gutierrez A, Ivanova E, Perna I, et al. 2007. Chromosomally unstable mouse tumours have genomic alterations similar to diverse human cancers. Nature 447: 966-971.

Motoyama $\mathrm{K}$, Inoue $\mathrm{H}$, Takatsuno $\mathrm{Y}$, Tanaka $\mathrm{F}$, Mimori $\mathrm{K}$, Uetake H, Sugihara K, Mori M. 2009. Over- and underexpressed microRNAs in human colorectal cancer. Int $J$ Oncol 34: 1069-1075.

Mulholland PI, Fiegler H, Mazzanti C, Gorman P, Sasieni P, Adams J, Jones TA, Babbage JW, Vatcheva R, Ichimura K, et al. 2006. Genomic profiling identifies discrete deletions associated with translocations in glioblastoma multiforme. Cell Cycle 5: 783-791.

Ota A, Tagawa H, Karnan S, Tsuzuki S, Karpas A, Kira S, Yoshida Y, Seto M. 2004. Identification and characterization of a novel gene, C13orf25, as a target for 13q31-q32 amplification in malignant lymphoma. Cancer Res 64: 30873095.

Rich JN. 2003. The role of transforming growth factor- $\beta$ in primary brain tumors. Front Biosci 8: e245-e260.

Richard S, Vogel G, Huot ME, Guo T, Muller WJ, Lukong KE. 2008. Sam 68 haploinsufficiency delays onset of mammary tumorigenesis and metastasis. Oncogene 27: 548-556.

Sachdeva M, Zhu S, Wu F, Wu H, Walia V, Kumar S, Elble R, Watabe K, Mo YY. 2009. p53 represses c-Myc through induction of the tumor suppressor miR-145. Proc Natl Acad Sci 106: 3207-3212.

Schumacher B, Hanazawa M, Lee MH, Nayak S, Volkmann K, Hofmann ER, Hengartner M, Schedl T, Gartner A. 2005. Translational repression of C. elegans p53 by GLD-1 regulates DNA damage-induced apoptosis. Cell 120: 357-368.

Sidman RL, Dickie MM, Appel SH. 1964. Mutant mice (Quaking and Jimpy) with deficient myelination in the central nervous system. Science 144: 309-311.

Stommel JM, Wahl GM. 2004. Accelerated MDM2 auto-degradation induced by DNA-damage kinases is required for p53 activation. EMBO J 23: 1547-1556.

Suzuki HI, Yamagata K, Sugimoto K, Iwamoto T, Kato S, Miyazono K. 2009. Modulation of microRNA processing by p53. Nature 460: 529-533.

Ule J, Jensen KB, Ruggiu M, Mele A, Ule A, Darnell RB. 2003. CLIP identifies Nova-regulated RNA networks in the brain. Science 302: 1212-1215.

Veeriah S, Taylor BS, Meng S, Fang F, Yilmaz E, Vivanco I, Janakiraman M, Schultz N, Hanrahan AJ, Pao W, et al. 2010. Somatic mutations of the Parkinson's disease-associated gene PARK2 in glioblastoma and other human malignancies. Nat Genet 42: 77-82.
Venkatraman ES, Olshen AB. 2007. A faster circular binary segmentation algorithm for the analysis of array CGH data. Bioinformatics 23: 657-663.

Ventura A, Young AG, Winslow MM, Lintault L, Meissner A, Erkeland SJ, Newman J, Bronson RT, Crowley D, Stone JR, et al. 2008. Targeted deletion reveals essential and overlapping functions of the miR-17 through 92 family of miRNA clusters. Cell 132: 875-886.

Vernet C, Artzt K. 1997. STAR, a gene family involved in signal transduction and activation of RNA. Trends Genet 13: 479484.

Volinia S, Calin GA, Liu CG, Ambs S, Cimmino A, Petrocca F, Visone R, Iorio M, Roldo C, Ferracin M, et al. 2006. A microRNA expression signature of human solid tumors defines cancer gene targets. Proc Natl Acad Sci 103: 22572261.

Wang Y, Lacroix G, Haines J, Doukhanine E, Almazan G, Richard S. 2010. The QKI-6 RNA binding protein localizes with the MBP mRNAs in stress granules of glial cells. PLoS ONE 5: e12824. doi: 10.1371/journal.pone.0012824.

Wesolowska A, Kwiatkowska A, Slomnicki L, Dembinski M, Master A, Sliwa M, Franciszkiewicz K, Chouaib S, Kaminska B. 2008. Microglia-derived TGF- $\beta$ as an important regulator of glioblastoma invasion-an inhibition of TGF- $\beta$-dependent effects by shRNA against human TGF- $\beta$ type II receptor. Oncogene 27: 918-930.

Wiedemeyer R, Brennan C, Heffernan TP, Xiao Y, Mahoney J, Protopopov A, Zheng H, Bignell G, Furnari F, Cavenee WK, et al. 2008. Feedback circuit among INK4 tumor suppressors constrains human glioblastoma development. Cancer Cell 13: $355-364$.

Wu J, Zhou L, Tonissen K, Tee R, Artzt K. 1999. The quaking I-5 protein (QKI-5) has a novel nuclear localization signal and shuttles between the nucleus and the cytoplasm. I Biol Chem 274: 29202-29210.

Wu JI, Reed RB, Grabowski PJ, Artzt K. 2002. Function of quaking in myelination: Regulation of alternative splicing. Proc Natl Acad Sci 99: 4233-4238.

Yan HL, Xue G, Mei Q, Wang YZ, Ding FX, Liu MF, Lu MH, Tang Y, Yu HY, Sun SH. 2009. Repression of the miR-17-92 cluster by $\mathrm{p} 53$ has an important function in hypoxia-induced apoptosis. EMBO I 28: 2719-2732.

Yin D, Ogawa S, Kawamata N, Tunici P, Finocchiaro G, Eoli M, Ruckert C, Huynh T, Liu G, Kato M, et al. 2009. Highresolution genomic copy number profiling of glioblastoma multiforme by single nucleotide polymorphism DNA microarray. Mol Cancer Res 7: 665-677.

Zearfoss NR, Clingman CC, Farley BM, McCoig LM, Ryder SP. 2011. Quaking regulates Hnrnpal expression through its 3' UTR in oligodendrocyte precursor cells. PLoS Genet 7: e1001269. doi: 10.1371/journal.pgen.1001269.

Zheng H, Ying H, Yan H, Kimmelman AC, Hiller DJ, Chen AJ, Perry SR, Tonon G, Chu GC, Ding Z, et al. 2008. p53 and Pten control neural and glioma stem/progenitor cell renewal and differentiation. Nature 455: 1129-1133. 


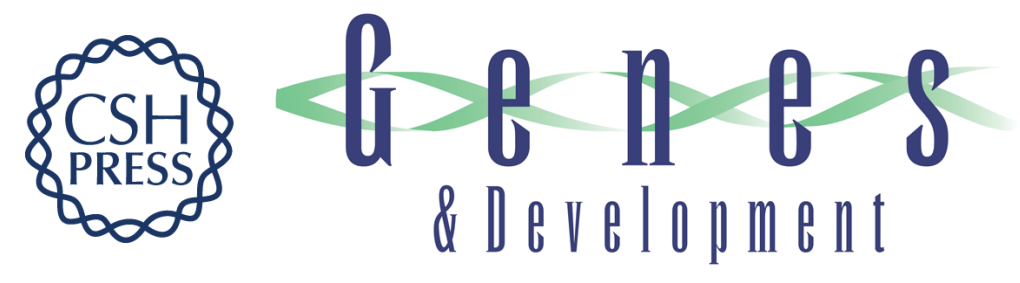

\section{STAR RNA-binding protein Quaking suppresses cancer via stabilization of specific miRNA}

An-Jou Chen, Ji-Hye Paik, Hailei Zhang, et al.

Genes Dev. 2012, 26:

Access the most recent version at doi:10.1101/gad.189001.112

Supplemental http://genesdev.cshlp.org/content/suppl/2012/07/02/26.13.1459.DC1
Material

References This article cites 56 articles, 19 of which can be accessed free at:

http://genesdev.cshlp.org/content/26/13/1459.full.html\#ref-list-1

License

Email Alerting Receive free email alerts when new articles cite this article - sign up in the box at the top

Service right corner of the article or click here.

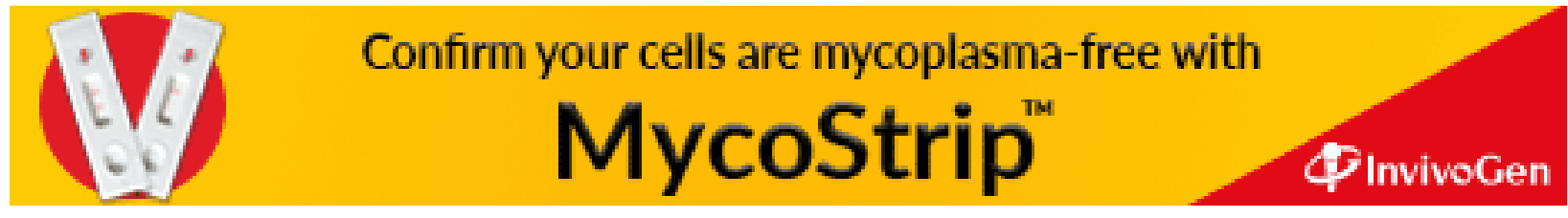

